\title{
A 28-Year-Old Woman with a Recent Onset of Painful Oral Erosions
}

Paul C. Edwards, BSc, MSc, DDS, $\operatorname{FRCD}(\mathrm{C})^{*}$,

Stephen Hess, BS, MS, DDS\#

Tarnjit Saini BDS, DDS $\uparrow$

Neil Norton, $\mathrm{PhD}^{* *}$

*Associate Professor, Dept. of Periodontics and Oral Medicine, School of Dentistry, University of Michigan, Ann Arbor, MI,

\# Associate Professor, Department of General Dentistry, Creighton University School of

Dentistry, Omaha, NE

ๆ Lt Col, US Army DENTAC, Brooke Army Medical Center, Fort Sam Houston, San Antonio, TX.

** Associate Professor of Oral Biology \& Pharmacology, Department of Oral Biology, Creighton University School of Dentistry, Omaha, NE

\section{ADDRESS CORRESPONDENCE AND REPRINT REQUESTS TO:}

Dr. Paul C. Edwards, Dept. of Periodontics and Oral Medicine, School of Dentistry, University of Michigan, 1011 N. University Avenue, Ann Arbor, MI 48109. E-mail: paulce@umich.edu

WORD COUNT: (not including Figure Legends):

KEY WORDS: clinical pathologic conference, histoplasmosis, vesiculo-erosive Final version published as:

Edwards, P. C., Hess, S., Saini, T., \& Norton, N. (2010). Twenty-Eight-YearOld Woman With Recent Onset of Painful Oral Erosions. Journal of Oral and Maxillofacial Surgery, 68(8), 1890-1895. doi:10.1016/j.joms.2009.06.040 


\section{Case Presentation:}

A 28-year-old single African-American woman presented to the dental emergency clinic at the Creighton University School of Dentistry, Omaha, Nebraska, with a chief complaint of painful ulcerations of the oral cavity. The lesions had been present for at least several weeks and apparently had been slowly increasing in severity over this period. The patient had previously presented to a walk-in medical clinic for evaluation and was initially prescribed amoxicillin. When no clinical improvement was noted, she was switched to Augmentin (amoxicillin and clavulanate), prescribed nystatin troches and chlorhexidine rinses and advised to see a dentist.

On initial presentation, the patient presented with multiple large crater-like erosions involving the palatal attached mucosa (figure 1a). The erosions were largely devoid of fibrin coating, except immediately along the border with the adjacent unaffected mucosa. In focal areas along the lateral margins of the erosions, an erythematous, raised, somewhat granular presentation was noted that resembled immature granulation tissue. The maxillary anterior attached gingiva had a somewhat granular appearance with linear ulcerations between the central incisors and interproximally between the left maxillary central and lateral incisors (figure 1b). The interdental papilla had a "punched out" necrotic appearance, but lacked a fetid odor. The palatal lesions were moderately painful to palpation. The patient denied having any similar vaginal or rectal lesions. The patient also reported that she had recently started to experience small pruritic erythematous skin lesions involving her arms (figure 2), legs and chest. Application of lateral pressure to the 
skin or oral mucosa failed to elicit a positive Nikolsky sign. No ocular or nail changes were noted.

The patient was somewhat concerned that the lesions in her mouth might be related to a sexually transmitted condition and volunteered that she had recently undergone screening for syphilis, gonorrhea, Chlamydia and HIV through a community clinic to rule out this possibility. The patient did report an infection with gonorrhea several years earlier that had been successfully treated. The remainder of her past medical history was unremarkable. The patient did not report dyspnea, cough, nausea or abnormal bowel movements, although she did report a several month history of increased fatigue and mild generalized malaise.

\section{Differential Diagnosis:}

Based on the several week history of multiple shallow erosions involving primarily the hard palate and the maxillary anterior facial attached gingiva in a young patient with a relatively uncomplicated medical history, the focus of our clinical differential diagnosis revolved around the most common causes of chronic erosive lesions in the oral cavity; namely immune-mediated conditions, systemic granulomatous processes and chronic infectious conditions. We were, of course, concerned about our patient's skin lesions and her history of mild malaise. However, it was not clear from her initial presentation whether the skin manifestations were a component of the disease process itself or were indicative of an allergic reaction to the amoxicillin. The history of mild generalized fatigue and malaise, while raising the possibility of hematological or systemic infectious 
processes, were, based on the patient's self-reported history, mild and non-specific and had apparently not increased in severity or otherwise changed over the preceding several weeks.

Our leading consideration was that this represented a chronic immune-mediated bullous disease. Pemphigoid, pemphigus, paraneoplastic pemphigus, linear IgA dermatosis, epidermolysis bullosa acquisita and chronic ulcerative stomatitis comprise a group of chronic conditions characterized by the production of auto-antibodies against various components of the epithelial/connective tissue attachment complex. These conditions affect the oral mucosa and/or skin and typically present with overlapping clinical presentations that often makes differentiation from each other based on clinical grounds alone difficult, particularly early in the disease process. Histopathological examination of both conventional formalin-fixed, paraffin-embedded hematoxylin and eosin (H\&E) stained tissue and tissue preserved in Michel's solution for immunofluorescent studies is usually necessary to arrive at a definitive diagnosis. Nevertheless, there are classical clinical differences between these conditions that warrant reviewing.

Mucous membrane pemphigoid is an immune-mediated mucocutaneous condition characterized by the presence of autoantibodies directed against various components of the basement membrane zone ${ }^{\mathrm{i}}$. In addition to the oral erosions, other mucosal sites including the nasal, vaginal, laryngeal and esophageal mucosa can be affected. Intact bullae are occasionally identified intra-orally. While the oral lesions typically heal without significant scarring, esophageal involvement can rarely lead to airway 
obstruction. More commonly, involvement of the ocular conjunctivae can lead to adhesions between the palpebral and bulbar conjunctivae and severe scarring. Skin lesions are uncommon.

Pemphigus vulgaris, resulting from the production of antibodies directed against protein components of the desmosomal attachment, desmoglein 1 and 3 , is seen with much less frequency than pemphigoid ${ }^{\mathrm{ii}}$. Oral lesions of pemphigus vulgaris, consisting of superficial erosions and ulcers that are distributed throughout the oral cavity but demonstrating preferential involvement of the palatal and labial mucosa, often precede the development of skin bullae by several months. Intact bullae are rarely noted intraorally. Ocular lesions may be seen, but these typically are not associated with the same degree of scarring noted in pemphigoid. A variant of pemphigus vulgaris, pemphigus vegetans, is characterized by the development of vegetative erosions $\mathrm{s}^{\mathrm{iii}}$, somewhat reminiscent of the exophytic areas noted at the periphery of several of the ulcerative lesions in our patient. Typically, however, the vermilion borders of the lips are involved preferentially.

Paraneoplastic pemphigus is a very rare variant of pemphigus that occurs in association with an underlying neoplasm, particularly non-Hodgkin's lymphomas, chronic lymphocytic leukemias and thymomas ${ }^{\text {iv }}$. The pathogenesis is poorly understood, although a percentage of cases may be related to tumor-associated overproduction of interleukin- 6 leading to the upregulation of anti-desmosomal antibody production. The clinical presentation is quite variable, ranging from classic pemphigus-like lesions, to pemphigoid-like ocular involvement to an erythema multiforme-like presentation with 
hemorrhagic crusting of the lips. In light of the patient's chronic low-grade malaise, this was a reasonable possibility, although still considered unlikely due to the rarity of this condition and the lack of an identified underlying neoplasm.

Several medications are known to cause either pemphigus ${ }^{\mathrm{v}}$ or pemphigoid-like ${ }^{\mathrm{vi}}$ eruptions (e.g. angiotensin-converting enzyme inhibitors, penicillamine) as well as non-specific ulcerations, also referred to as "stomatitis medicamentosa" (e.g. hydroxyurea, nicorandil, indomethacin, methotrexate). Obviously, review of the patient's medical history would identify potential offending drugs, none of which were indicated in our patient.

Linear IgA dermatosis (linear IgA disease; linear IgA bullous dermatosis of childhood), epidermolysis bullosa acquisita and chronic ulcerative stomatitis are rare cause of chronic erosive lesions of the oral cavity. Linear IgA dermatosis affects both children and adults and primarily involves the dermis ${ }^{\mathrm{vii}}$. It is characterized by the development of large clear to hemorrhagic bullae occurring on normal or erythematous skin. In contrast to mucous membrane pemphigoid, oral lesions in the absence of extensive skin involvement are uncommon in linear IgA dermatosis. Epidermolysis bullosa acquisita is a chronic autoimmune blistering disease of the skin that in some cases can present with predominant mucosal involvement that is often clinically indistinguishable from mucous membrane pemphigoid ${ }^{\text {viii }}$. Chronic ulcerative stomatitis (CUS) is another rare immunemediated erosive condition that primarily affects the tongue, buccal mucosa and gingiva $^{\text {ix }}$. The clinical presentation of CUS is highly variable. Specialized 
immunofluorescent studies are typically necessary to differentiate between these conditions.

Erythema multiforme is typically a self-limiting acute process characterized by the abrupt onset of rapidly progressing erosions of the lip, tongue, gingiva and lip mucosa coupled with erythematous skin macules that form "target-like" lesions ${ }^{\mathrm{x}}$. The oral lesions typically start as small vesicles that quickly rupture, leaving a raw ulcerated pseudomembranous surface. Viral-like prodromal symptoms ranging from generalized malaise to fever and cough are often noted. In approximately half of the cases, no precipitating factor is identified. In the remaining cases, common triggers include recurrent herpes activation and drugs such as antibiotics and non-steroidal antiinflammatory drugs. Typically, the lesions will resolve in 2-3 weeks, although recurrent episodes may be seen, especially in those cases related to herpes virus reactivation.

Hematological conditions such as agranulocytosis, aplastic anemia and acute leukemia can cause generalized erosions of the oral cavity, but their onset tends to be more acute and fulminant compared to the relatively prolonged history in our patient's case.

While the possibility that these lesions represented major aphthous ulceration was briefly considered, the clinical presentation was not typical ${ }^{\mathrm{xi}}$. Classically, in the immunocompetent patient, major aphthae develop on the unattached gingiva, primarily involving the soft palate/tonsillar pillae or floor of the mouth. A well-defined, thin erythematous border is typically present, and the degree of pain is usually extreme. While 
atypical aphthous-like ulcerations are relatively common in patients with acquired immunodeficiency syndrome (AIDS), our patient insisted that she was HIV negative. In addition, the clinical appearance of the anterior maxillary attached gingiva was not compatible with major aphthae, having a slightly granular appearance.

This granular presentation on the maxillary buccal gingiva raised the possibility of an underlying granulomatous process, most likely systemic considering the widespread involvement. Chronic granulomatous disease, a rare congenital condition associated with functional defects of phagocytes characterized by recurrent episodes of bacterial and fungal infections that are often life-threatening, was not seriously considered in our differential diagnosis because it typically manifests within the first few years of life. Wegener's granulomatosis is an uncommon chronic condition of unknown etiology characterized by the development of necrotizing granulomatous inflammation that affects the upper and lower respiratory tract, as well as vasculitis of the small to medium diameter blood vessels. Patients typically present with non-specific constitutional symptoms, chronic sinonasal involvement and various cutaneous manifestations. Oral involvement is rare ${ }^{\mathrm{xii}}$. The characteristic intraoral appearance, consisting of a bumpy, granular, hemorrhagic mucosal surface primarily affecting the buccal gingival surface, is termed "strawberry gingivitis." In more advanced cases, irregular erosions and ulcers resembling the palatal lesions seen in our patient may be noted, potentially affecting any mucosal surface. 
Acute infectious processes such as primary herpetic gingivostomatitis could be ruled out based on the prolonged duration of the lesions. The possibility of AIDS-associated atypical ulcerations associated with recurrent herpes should be included in the differential diagnosis, even in light of the patient's purported, but unconfirmed, HIV-negative status.

Another consideration was that the oral and skin lesions represented mucocutaneous manifestations of secondary syphilis. However, the "mucous patches" of secondary syphilis present more typically as multiple edematous irregular appearing white thickened plaques, although epithelial necrosis of these plaques may lead to the formation of sizeable ulcers ${ }^{\text {xiii }}$. Non-specific constitutional symptoms such as malaise and weight loss are common. Diffuse erythematous skin rashes are a consistent presentation, but these are typically larger than the lesions noted in our patient. The patient's claim that she had recently tested negative for a number of sexually transmitted diseases, including syphilis, should rule this out.

Tuberculosis affecting the oral cavity is extremely rare ${ }^{\text {xiv }}$. Presumably oral lesions develop via inoculation from coughed up pulmonary organisms or following hematogenous spread. Oral lesions present as large ulcer with a central vegetative/granulomatous appearance with a slightly indurated border. Cervical lymphadenopathy is common. Multiple lesions, as noted in this case, would be extremely uncommon absent disseminated extrapulmonary disease in a severely immunocompromised patient. 
Although uncommon, invasive systemic mycoses such as mucormycosis, aspergillosis, histoplasmosis, cryptococcus, and North American blastomycosis can present with a clinical appearance similar to that seen in our patient. Typically, these are associated with some type of underlying condition leading to a weakened immune response.

Finally, the presence of the blunted interproximal papillae seen in our patient could raise the consideration of necrotizing ulcerative gingivitis (NUG), an acute condition characterized by spirochete-mediated destruction of the interdental papillae resulting in a "punched out" appearance. However, the prolonged duration and the presence of large palatal erosions are not consistent with NUG.

\section{Diagnosis and Management:}

An incisional biopsy was obtained from the attached palatal mucosa under local anesthesia, and the tissue placed in formalin and submitted for hematoxylin and eosin staining. Although we were suspicious of an immune-mediated vesiculobullous process, we elected not to obtain additional tissue for direct immunofluorescence at this stage since our differential diagnosis was quite broad.

Histologic examination revealed oral mucosa surfaced by intact parakeratinized stratified squamous epithelium overlying variably dense fibrovascular connective tissue. A moderately dense mixed inflammatory cell infiltrate was noted within the deeper connective tissue along one of the edges of the specimen (figure 3a). On close examination of the H\&E-stained specimen, faint round to ovoid intracytoplasmic bodies 
were noted within macrophages along one of the lateral aspects of the specimen (figure 3b). These intracytoplasmic bodies were better visualized following periodic acid-Schiff (PAS; figure 3c) and Gomori methenamine silver (GMS; figure 3d) staining. These fungal organisms, measuring approximately $2-4 \mu \mathrm{m}$ in diameter, consisting of a central round basophilic body surrounded by a clear staining halo under H\&E staining, were consistent with Histoplasma spp. Definitive identification of H. capsulatum, either by testing of cultured tissue with a chemiluminescent DNA probe or serologic/urine enzyme immunoassay screening for Histoplasma polysaccharide antigen, was not performed. However, the histopathologic presentation was strongly suggestive of Histoplasma capsulatum.

The final diagnosis was intrahistiocytic fungal infection consistent with histoplasmosis.

Several attempts were made to contact the patient by telephone in order to refer her to a local infectious disease clinic. Eventually, a family member volunteered that the patient had been admitted to a neighboring hospital the day after her biopsy had been performed for treatment of "disseminated fungal disease." Further attempts at follow up were unsuccessful.

\section{Discussion:}

Histoplasmosis is a systemic invasive fungal disease endemic to the parts of the Midwest of the United States, in particular around the Mississippi and Ohio Valley, as well as in parts of the Central and Southeast states. In the Americas, histoplamosis is caused by the 
dimorphic yeast histoplasma capsulatum var. capsulatum, which grows in nitrogen-rich soil as well as in areas contaminated with bird and bat feces. At 37 degrees Celsius, the mold converts to a 2 to 4 um oval yeast form that is taken up by macrophages in the lung. An estimated $80 \%$ of the adult population in parts of the Midwest show immunologic evidence of prior exposure to the organism.

Histoplasma exposure can result in acute or chronic forms of histoplasmosis. Primary acute pulmonary histoplasmosis is a self-limiting form that infects up to 500,000 people per year in the United States. The vast majority of infected people are not even aware they have been infected, especially when the individual has been exposed to only small numbers of spores ${ }^{\mathrm{xv}}$. When exposed to very high numbers of spores, individuals are more likely to develop acute pulmonary histoplasmosis, manifesting as non-specific pulmonary symptoms such as cough, and dyspnea as well as constitutional symptoms ranging from low-grade fever to malaise that are often misinterpreted as a routine upper respiratory tract infection. In the immunocompetent host, this is usually self-limiting and often does not require treatment.

Individuals at the greatest risk of developing chronic histoplasma infection include those with suppressed immune systems and/or underlying chronic lung disease. Chronic pulmonary infection is associated with the development of pulmonary cavitations and is characterized by pulmonary signs and symptoms reminiscent of tuberculosis. This presentation is primarily seen in patients with chronic obstructive lung disease. If untreated, the long-term outcome invariably involves progressive pulmonary 
insufficiency and death. There is also a rare disseminated form in which spores spread to the lymph nodes, bone marrow, liver, spleen, adrenal glands and gastrointestinal tract, resulting in widespread symptoms of systemic infection, lymphadenopathy, hepatosplenomegaly and other extrapulmonary manifestations.

The vast majority of cases of oral histoplasmosis are associated with the disseminated form of the disease ${ }^{\mathrm{xvi}}$. The most common oral sites of involvement include the tongue, palate, gingiva and buccal mucosa ${ }^{\text {xvii }}$. Oral lesions classically have an irregular ulcerated appearance with a granular exophytic margin and rolled indurated borders resembling squamous cell carcinoma ${ }^{\text {xviii }}$. Erosion of underlying bone may be seen. However, the clinical presentation is highly variable. Multiple lesions, as in our case, are uncommon. Facial skin lesions, occasionally resembling the skin papules associated with molluscum contagiosum, may also be present.

The disseminated form of histoplasmosis, the underlying type seen in patients presenting with oral manifestations, is usually seen only in immunocompromised patients, either secondary to AIDS (especially with CD4 counts of less than 150 cells/um), immunosuppressive therapy or severe debilitation/advanced age. Prior to the AIDS epidemic, disseminated histoplasmosis was extremely rare. Since the introduction of effective anti-retroviral drugs, this has once again become less common. Patients treated with biologic agents that target tumor necrosis factor-alpha, such as infliximab (Remicade; Centocor, Malvern, PA), adalimumab (Humira; Abbott Laboratories, Abbot Park, IL) and etanercept (Enbrel; Amgen Corp., Thousand Oaks, CA), also appear to be 
at a substantially increased risk of developing invasive fungal infections, particularly histoplasmosis ${ }^{\mathrm{xix}, \mathrm{xx}}$. To date, the majority of these cases have occurred among patients living in areas endemic for histoplasmosis who were also receiving additional immunosuppressant therapy. In one study ${ }^{\mathrm{xx}}$, the death rate in patients developing disseminated fungal infection following tumor necrosis factor-alpha blockade therapy approached $20 \%$.

Our patient denied a history of AIDS or immunosuppressive therapy, although without direct testing, her HIV status and CD4 count could not be definitely determined. Interestingly, it has been demonstrated ${ }^{\mathrm{xxi}}$ in a murine model that methamphetamine abuse reduces the immune response to histoplasma infection through several mechanisms. Methamphetamine use is widespread in parts of the Midwest of the United States, including Nebraska ${ }^{\mathrm{xxii}}$. Since we never asked our patient specifically about any history of drug abuse, we can only speculate in retrospect as to whether methamphetamine abuse might have been a contributing factor in this case.

Treatment of disseminated histoplasmosis involves management of any underlying disease process, such as antiretroviral therapy to increase the CD4 count in AIDS, combined with long-term systemic antifungal therapy. The recommended therapy for mild to moderately severe disseminated histoplasmosis is $200 \mathrm{mg}$ oral itraconazole twice daily for 6 to 18 months, depending on response to therapy and underlying host resistance $^{\mathrm{xv}}$. In cases of severe disseminated histoplasmosis, an initial short course with amphotericin B, switching to itraconazole as the patient's condition improves, is 
suggested. Long term prophylaxis with itraconazole, 200mg per day, should be considered in AIDS patients with CD4 counts below 150 to 200 cells/um. 


\section{Captions to Figures:}

Figure 1a:

A 28-year-old woman presented with a several week history of large painful erosions involving the palatal attached mucosa. The erosions are largely devoid of fibrin coating. Along the lateral margins of the erosions, a somewhat granular appearance resembling granulation tissue is noted.

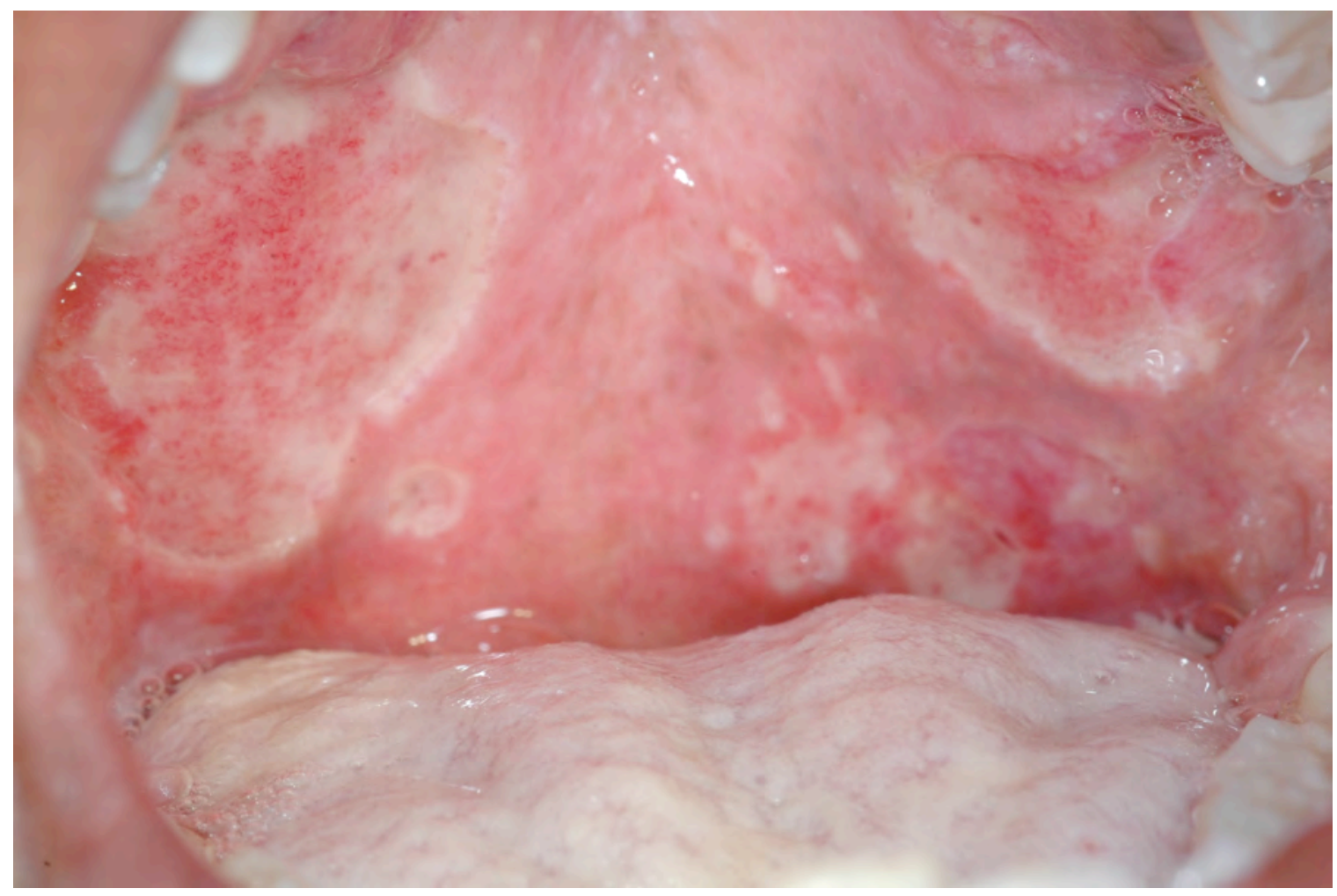


Figure 1b:

The maxillary anterior attached gingiva exhibits a granular appearance with linear ulcerations noted interproximally between the central incisors and between the left maxillary central and lateral incisors The interdental papilla has a "punched out" necrotic appearance.

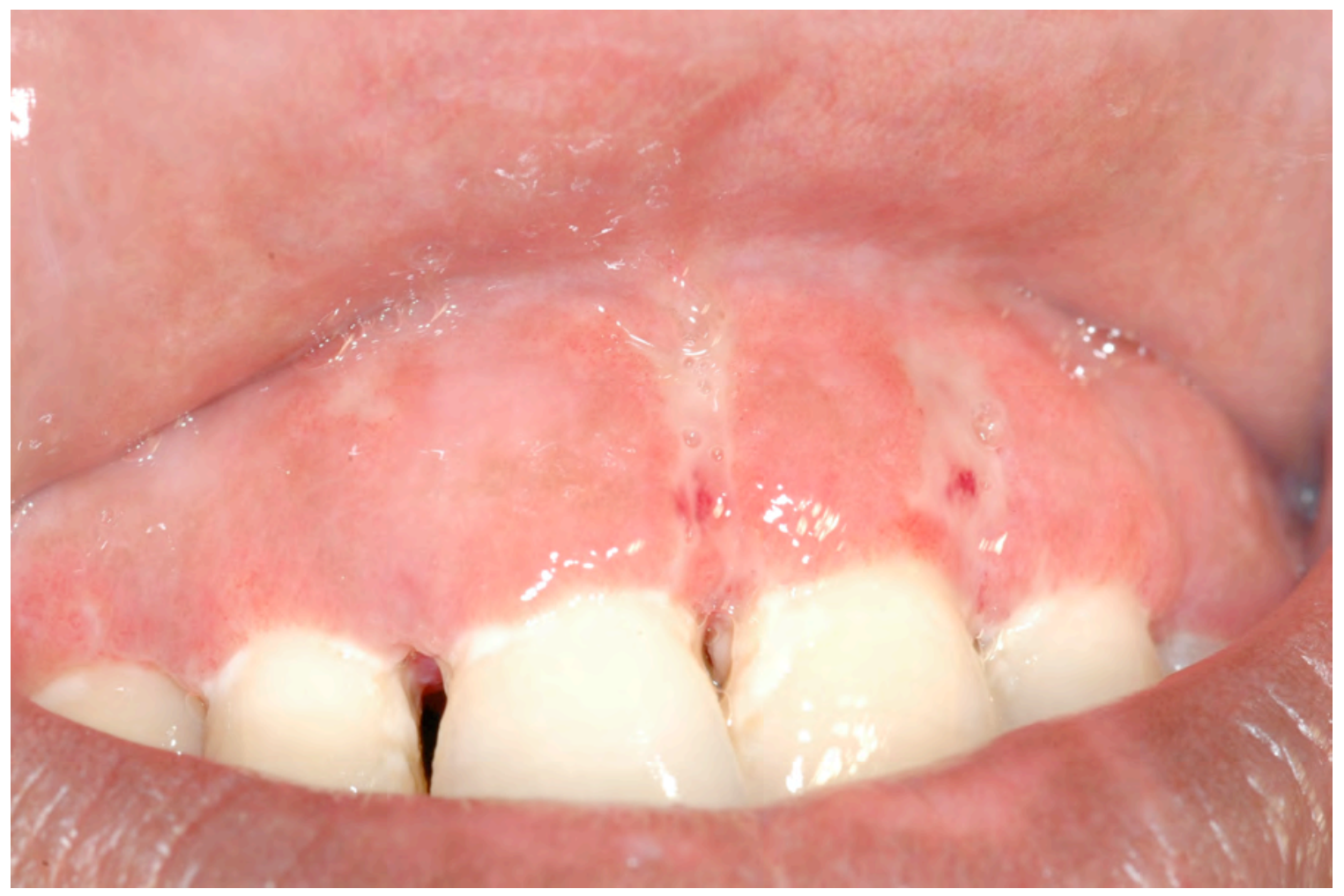




\section{Figure 2:}

The patient recently started to experience small pruritic erythematous skin lesions involving her arms, legs and chest. A closeup of the skin lesions on her arm is shown.

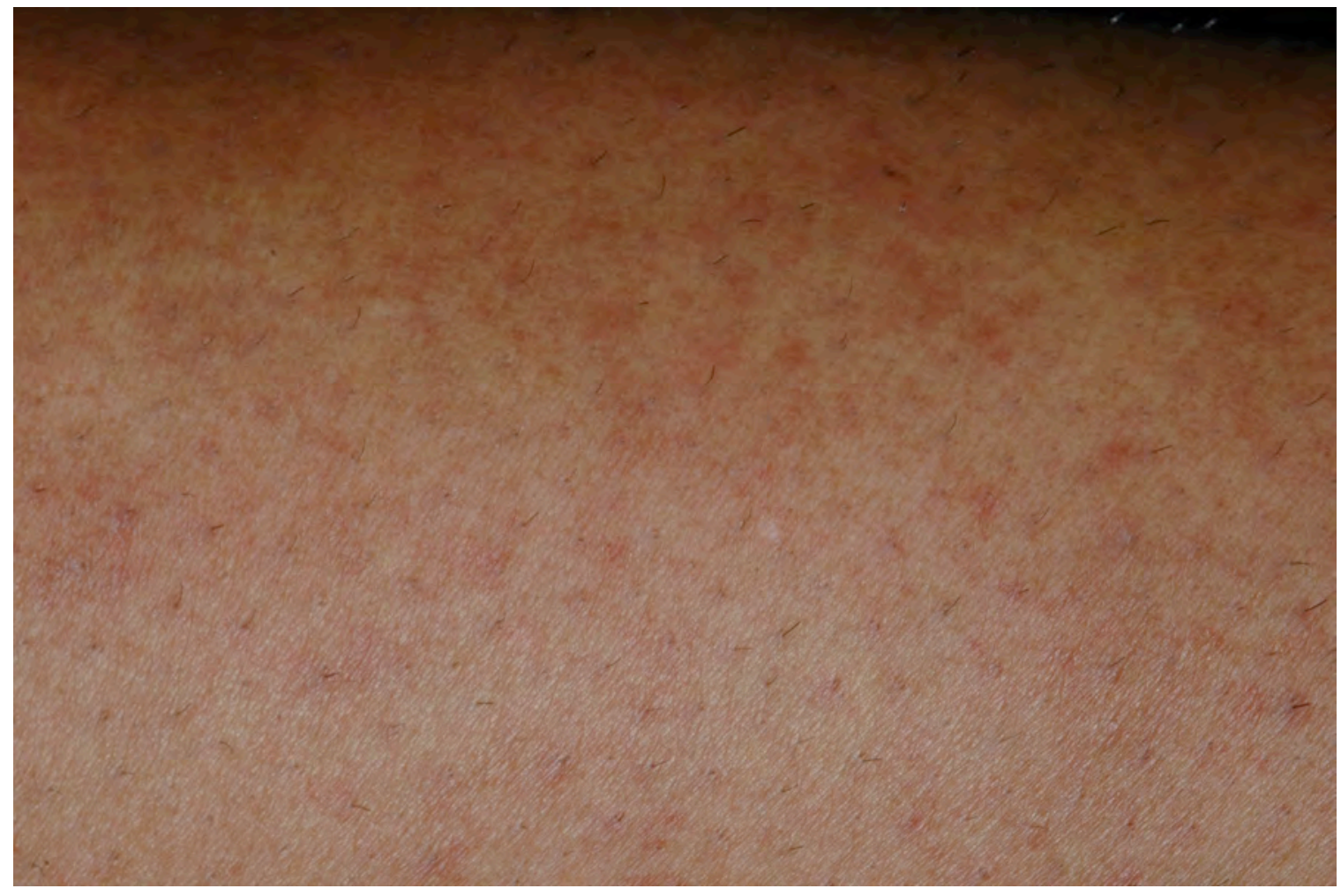


Figure 3a:

Histologic examination reveals oral mucosa covered by intact epithelium containing a moderately dense mixed inflammatory cell infiltrate along the lower edge of the specimen (hematoxylin and eosin, original magnification 40x).

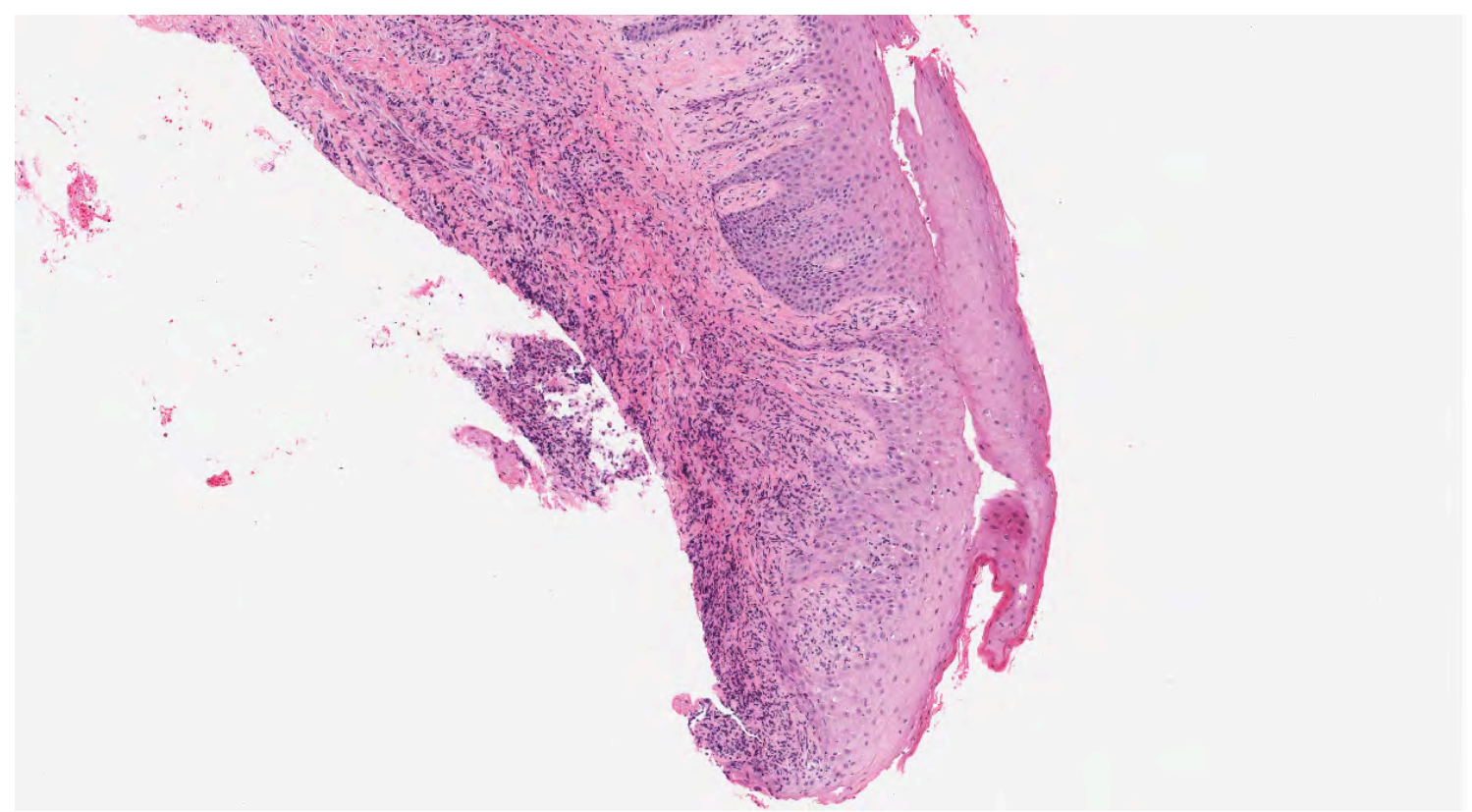


Figure 3b:

On higher magnification, numerous faint round to ovoid intracytoplasmic bodies consisting of a central round basophilic body surrounded by a clear staining halo can be visualized within macrophages (hematoxylin and eosin, original magnification 200x).

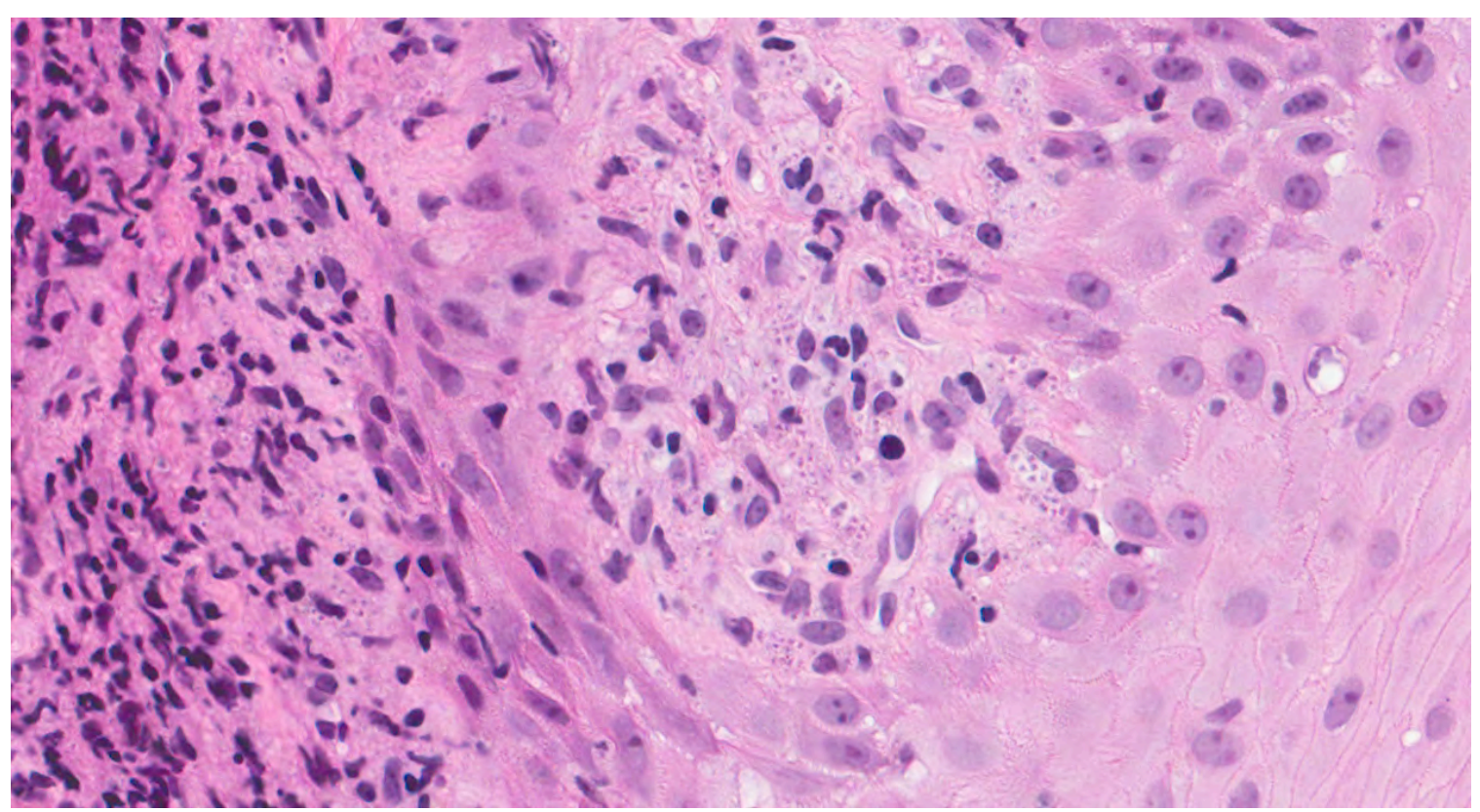


Figure 3c:

These fungal organisms, measuring approximately $2-4 \mu \mathrm{m}$ in diameter, are better visualized following periodic acid-Schiff(PAS) staining (original magnification 200x).

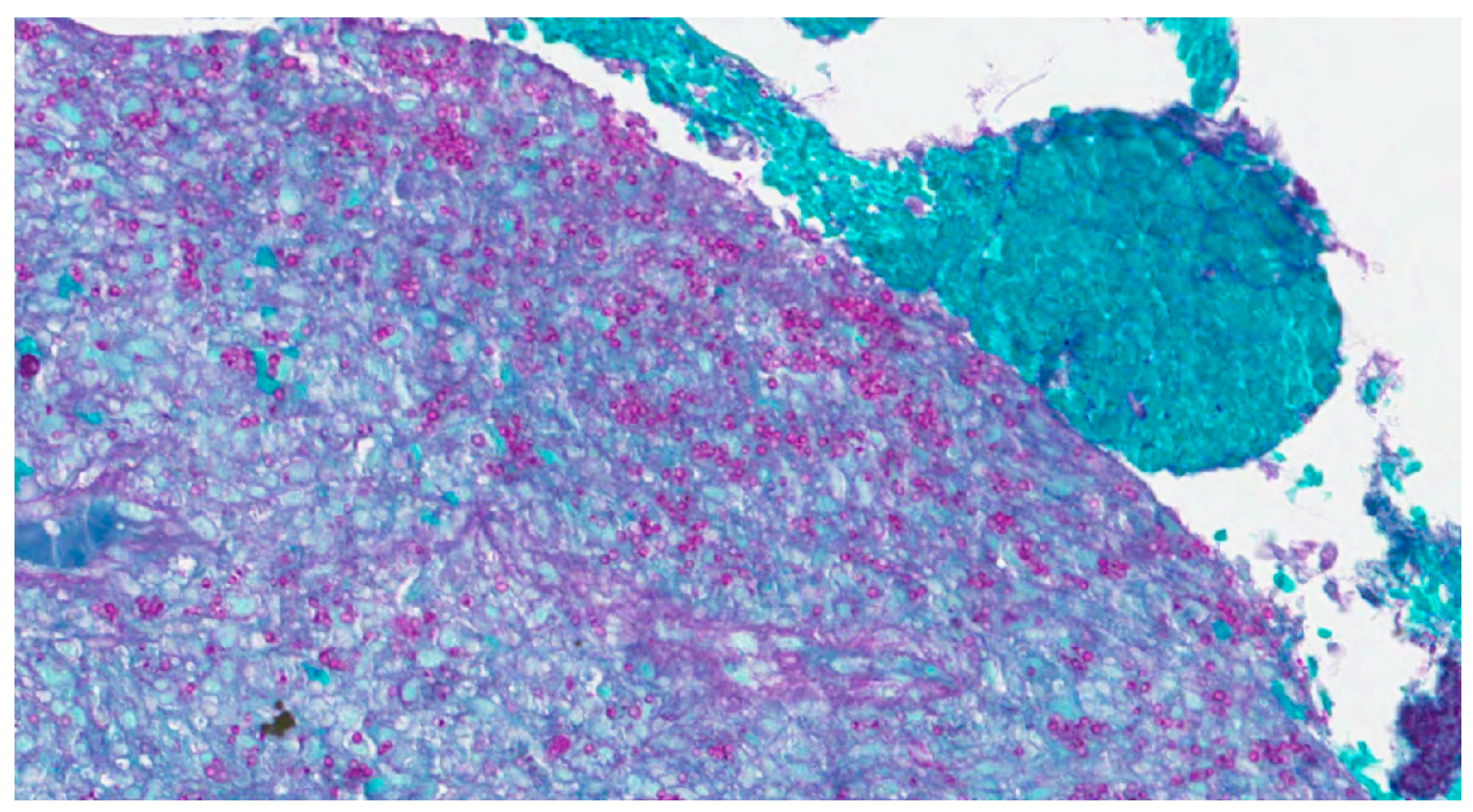


Figure 3d:

Gomori methenamine silver (GMS) stain of the same section of tissue (original magnification 200x).

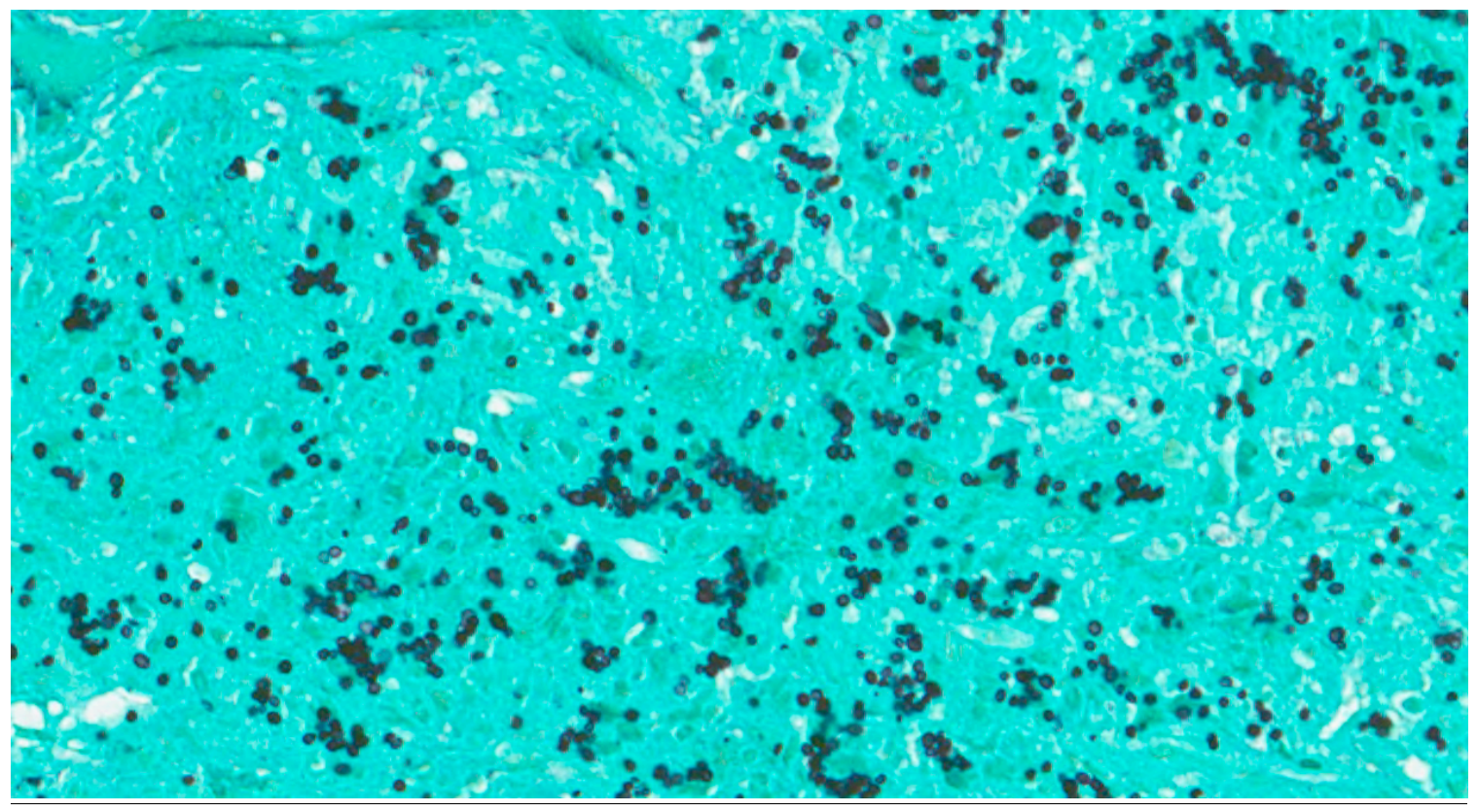




\section{References}

${ }^{\mathrm{i}}$ Scully C, LoMuzio L. Oral mucosal diseases: mucous membrane pemphigoid. Brit J Oral Maxillofac Surg 2008;46:358-66.

${ }^{\text {ii }}$ Scully C, Mignogna M. Oral mucosal diseases: pemphigus. Brit J Oral Maxillofac Surg $2008 ; 46: 272-7$.

iii Markopoulos AK, Antoniades DZ, Zaraboukas T. Pemphigus vegetans of the oral cavity. Int J Dermatol 2006;45:425-8.

${ }^{\text {iv }}$ Zhu X, Zhang B. Paraneoplastic pemphigus. J Dermatol 2007;43:503-11.

${ }^{v}$ Brenner S, Bialy-Golan A, Ruocco V. Drug-induced pemphigus. Clin Dermatol 1998;16:393-7.

${ }^{\text {vi }}$ Vassileva S. Drug-induced pemphigoid: bullous and cicatricial. Clin Dermatol 1992;16:379-87.

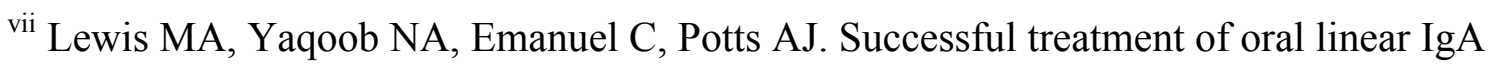
disease using mycophenolate. Oral Surg Oral Med Oral Pathol Oral Radiol Endod 2007;103:483-6.

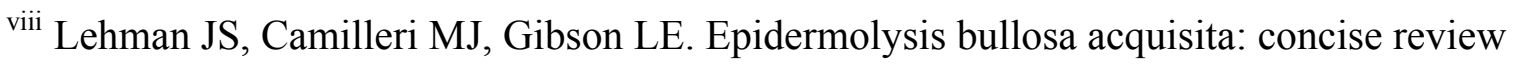
and practical considerations. Int J Dermatol 2009;48:227-36.

ix Solomon LW, Aguirre A, Neiders M, Costales-Spindler A, Jividen Jr GJ, Zwick MJ, Kumar V. Chronic ulcerative stomatitis: Clinical, histopathologic, and immunopathologic findings. Oral Surg Oral Med Oral Pathol Oral Radiol Endod 2003;96:718-26.

${ }^{x}$ Al-Johani KS, Fedele S, Porter SR. Erythema multiforme and related disorders. Oral Surg Oral Med Oral Pathol Oral Radiol Endod 2007;103:642-54. 


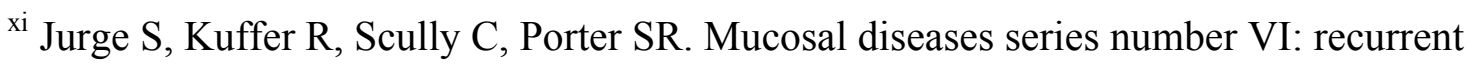
aphthous stomatitis. Oral Diseases 2006;12:1-21.

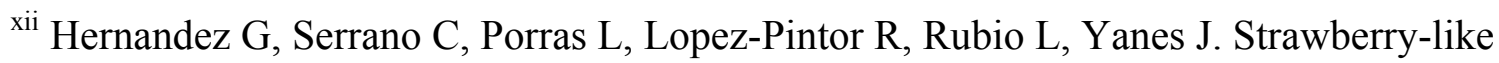
gingival tumor as the first sign of Wegener's granulomatosis. J Periodontol 2008;79:1297-1303.

xiii Little JW. Syphilis: an update. Oral Surg Oral Med Oral Pathol Oral Radiol Endod 2005;100:3-9.

${ }^{\text {xiv }}$ Hale RG, Tucker DI. Head and neck manifestations of tuberculosis. Oral Maxillofac Surg Clin North Am 2008;20:635-42.

${ }^{\mathrm{xv}}$ Kauffman CA. Histoplasmosis: a clinical and laboratory update. Clin Micrbiol Rev 2007;20:115-32.

${ }^{\text {xvi }}$ Chinn H, Chernoff DN, Migliorati CA, Silverman Jr S, Green TL. Oral histoplasmosis in HIV-infected patients: a report of two cases. Oral Surg Oral Med Oral Pathol Oral Radiol Endod 1995;79:10-4.

xvii Bhagwat PV, Hanumanthayya K, Tophakhane RS, Rathod RM. Two unusual cases of histoplasmosis in human immunodeficiency virus-infected individuals. Indian Dermatol Venereol Leprol 2009;75:173-6.

xviii Narayana N, Gifford R, Giannini P, Casey J. Oral histoplasmosis: an unusual presentation. Head Neck 2009;31:P274-7.

${ }^{\text {xix }}$ Furst DE, Wallis R, Broder M, Beenhouwer DO. Tumor necrosis factor antagonists: different kinetics and/or mechanisms of action may explain differences in the risk for developing granulomatous infection. Semin Arthritis Rheum 2006;36:159-67. 
${ }^{\mathrm{xx}}$ Tsiodras S, Samonis G, Boumpas DT, Kontoyiannis DP. Fungal infections complicating tumor necrosis factor alpha blockade therapy. Mayo Clin Proc 2008;83:181-94.

${ }^{x x i}$ Martinez LR, Mihu MR, Gacser A, Santambrogio L, Nosanchuk JD. Methamphetamine enhances histoplasmosis by immunosuppression of the host. J Infectious Diseases 2009;200:131-41.

xxii Shaner JW, Kimmes N, Saini T, Edwards PC. Meth mouth: rampant caries in methamphetamine abusers. AIDS Patient Care and STDs 2006;20:4-8. 